\title{
FORO
}

Recibido 18.02.2013. Aprobado 18.06.2013

Evaluado por el sistema double blind review. Editor Científico: Mário Aquino Alves, Ezequiel Reficco y Juan Arroyo

DOI: http://dx.doi.org/10.1590/So034-759020140107

\section{AGREGANDO VALORES A LAS CADENAS DE VALOR}

\section{Agregando valores às cadeias de valor}

\section{Adding values to value chains}

\section{RESUMEN}

Este artículo presenta una matriz de factores a tomar en cuenta para priorizar las cadenas de valor agrícolas seleccionadas como foco de las iniciativas público-privadas para impulsar su desarrollo. Dichos factores dan especial importancia a temas de responsabilidad social empresarial. Se ilustra su uso con datos y una revisión de la literatura sobre Perú.

PALABRAS CLAVE / Agronegocios, responsabilidad social empresarial, sostenibilidad, alianzas públicas-privadas y pobreza.

\section{ABSTRACT}

This article presents a matrix of factors to prioritize agricultural value chains targeted for public-private initiatives intended to foster their further development. The factors give noteworthy attention to issues related to corporate social responsibility. Data analysis and a literature review serve to illustrate the use of the factors in the case of Peru.

KEYWORDS / Agribusiness, corporate social responsibility, public-private alliances, sustainability, poverty.

\section{RESUMO}

\section{GREGORY J. SCOTT}

gscott@pucp.pe

Profesor del CENTRUM Graduate Business School, Pontificia Universidad Católica del Perú, Lima - Perú
Este artigo apresenta uma matriz de fatores a levar em conta para priorizar as cadeias de valor agrícolas selecionadas como foco das iniciativas público-privadas para impulsionar o seu desenvolvimento. Tais fatores conferem especial importância a temas de responsabilidade social empresarial. Seu uso é ilustrado com dados e uma revisão da literatura sobre o Peru.

PALAVRAS CHAVE | Agronegócios, responsabilidade social empresarial, sustentabilidade, alianças público-privadas, pobreza. 


\section{INTRODUCCIÓN}

Las últimas tres décadas han sido testigos de una serie de cambios profundos en el ámbito empresarial en Latinoamérica. La privatización, liberalización de los mercados domésticos e implementación de los Tratados de Libre Comercio en Latinoamérica (Anderson \& Valenzuela, 2010) han resultado en una reducción de la participación del sector público en la economía y un perfil más notable de las empresas privadas en la misma. Estos eventos han contribuido a la generación de lo que algunos observadores han caracterizado como una nueva relación entre la empresa y la sociedad en América Latina (Reficco \& Ogliastri, 2009). Dicha relación implica involucrar a la empresa en actividades de responsabilidad social empresarial (RSE) las cuales van más allá de simplemente maximizar las utilidades, a fin de incluir el buen gobierno corporativo, la sostenibilidad del medio ambiente, promover los negocios inclusivos - hasta dar inicio a una nueva manera de administrar una empresa (Flores, Ogliastri, Peinado-Vara, \& Petry, 2007).

Entonces entrando el siglo XXI hay un renovado interés en la relación entre la producción agrícola y su eventual uso final como tierra fértil para aprovechar nuevas oportunidades comerciales al nivel nacional, regional e internacional (Reardon \& Timmer, 2012). Están vistas también como posibles maneras de reducir la pobreza rural, atender a la vez las exigencias de los consumidores urbanos (Wilkinson \& Rocha, 2009) e inclusive ayudar en conservar el medio ambiente (Ickis, Leguizamón, Metzger, \& Flores, 2009). Dadas estas circunstancias, han crecido múltiples instancias tanto a nivel empresarial (Gómez, 2008; Leguizamón, Petry, \& Hernández, 2007) como nacional (Espinal \& Martínez, 2006) y hasta regional (Piñones Vázquez, Acosta Avila, Tartanac, 2006), en dar mayor énfasis en mejorar la competitividad de lo que constituye la cadena de valor (CDV).

En el sector agronegocios, la CDV se refiere a la serie de procesos y/o procedimientos que ocurren después de la cosecha de un cultivo hasta la entrega para su uso final. No obstante, las CDVs agrícolas operan en el contexto de un cultivo, empresa, organización (p.ej., ONG, gremio), con diferentes fines o actores (Bolwig, Du Toit, Riisgaard, \& Halberg, 2008). Más aún, no necesariamente incorporan actividades a la RSE, por ejemplo, como disminuir la pobreza rural o conservar el medio ambiente (Drost, Van Wijk, \& Vellema, 2011). Entre las empresas que vienen practicando la RSE en Latinoamérica (Tromben, 2011), la incidencia de agronegocios es notable (Fernández, Trujillo, \& Gutiérrez, 2003; Leguizamón \& Ickis, 2005; Lozano, Romero, \& Serrano, 2003).

El impulso de la RSE ha sido un fenómeno relativamente reciente en el mundo empresarial Latinoamericano (Austin \&
Chu, 2006) en parte como una respuesta a los cambios macroeconómicos ya mencionados. No obstante, todavía no hay un consenso en cómo ir más allá de las prácticas particulares de algunas empresas en temas de la RSE para explotar el potencial del sector privado para desarrollar dichas iniciativas en las CDVs. De todas maneras, dada la evolución de la relación empresa-sociedad, existe un creciente interés en vincular esfuerzos públicos con empresariales en lo referido a fomentar la RSE en las CDVs como parte de una estrategia de mejorar su competitividad.

El resto del artículo presenta primero un breve resumen de la evolución de la RSE en América Latina. Después se analiza la transformación de los sistemas alimenticios en la región y los estudios sobre las CDVs relacionados con ella. Posteriormente se presenta una matriz de factores para identificar los productos y lugares con el fin de focalizar las actividades relacionadas con la colaboración público-privada para desarrollar las CDVs incluyendo la RSE. Se ilustra su uso en el caso con una revisión de cultivos y temas territoriales en el caso del Perú. El artículo concluye con algunas observaciones finales.

\section{LITERATURA}

Según algunos observadores, la RSE en América Latina tiene sus orígenes en los Estados Unidos al final del siglo XIX (D`Alessio \& Marquina, $s / f)$. Todo comenzó cuando magnates de la época decidieron proporcionar servicios comunitarios a sus trabajadores. Luego junto con el crecimiento y expansión de las operaciones de las empresas multinacionales en los años 70 y 80 en los países en vía de desarrollo, los temas de medio ambiente, los derechos de los consumidores y la lucha contra la pobreza, entre otros, empezaron de captar mayor atención en América Latina. Las nuevas ideas gerenciales que surgieron después (Lozano, Romero, \& Serrano, 2003) estuvieron impulsados por las políticas macroeconómicas de apertura, la larga tradición de filantropía de ciertas empresas en la región (González \& Márquez, 2005), y entre otras iniciativas internacionales el Reporte Brundtland (Wced, 1987) de las Naciones Unidas. Dicho informe lanzó el concepto del "desarrollo sostenible" o aquel que promueve el crecimiento económico hoy sin perjudicar las posibilidades de futuras generaciones. En este espíritu, el informe hizo una llamada tanto a los gobiernos como a las empresas de incorporar dicha consideración en sus operaciones. También se abrió el camino para la primera Conferencia de las Naciones Unidas sobre medio ambiente llevado a cabo en Rio de Janeiro en 1992 para establecer metas al respecto. Con tiempo el concepto de desarrollo sostenible fue evolucionando para incorpo- 
rar además de la necesidad económica, las de medio ambiente y social a la vez. De esta manera, se fue emergiendo la teoría de la "comunidad vinculada", o sea la importancia del grupo o individuo que puede afectar las actividades de la empresa pero también incluye aquellos que pueden estar afectados por estas. Entrando al siglo XXI, las empresas como sus actividades de RSE van recibiendo mucho más monitoreo que antes catalizando a la vez la formación de organizaciones nacionales dedicadas no solo al monitoreo, sino también a redes regionales de apoyo, promoción y evaluación (Reficco \& Ogliastri, 2009).

Hay tres aspectos sobresalientes del avance de RSE en América Latina en las últimas décadas. Primero, hay una consolidación del creciente número de temas o áreas de iniciativas operacionales bajo la sombrilla de la RSE (Flores, Ogliastri, Peinado-Vara, \& Petry, 2007). Ellos son: la conservación del medio ambiente, el desarrollo de la comunidad, asuntos de consumidores y clientes, prácticas internas laborales, prácticas frente a los proveedores y distribuidores, derechos humanos, y gobierno corporativo (véase, p.ej., Perú 2021, 2010). Segundo, la RSE se refiere a actividades voluntarias por parte de la empresa que se basa en un fuerte compromiso hacia el dialogo. Juntos se apuntan a la búsqueda de beneficios para la sociedad además de la empresa, y con mayor frecuencia involucran coordinación, sino alianzas con entes del gobierno (Flores, Ogliastri, Peinado-Vara, \& Petry, 2007). Tercero, no hay una definición única de la RSE (Reficco \& Ogliastri, 2009). Desde el punto de vista gerencial, la idea de la RSE va desde un costo para hacer negocios en determinado sitio - o en otras palabras una nueva forma de filantropía corporativa - hasta que esta representa una nueva manera de sostener la competitividad de una empresa (Flores, Ogliastri, Peinado-Vara, \& Petry, 2007; Tromben, 2011). En ese sentido, hay una inclinación de fijarse más en la parte operacional del concepto y su relevancia para el desarrollo de la empresa y la sociedad que una definición más precisa en sí.

En la parte operacional de la RSE, cada uno de los temas tiene diferentes dimensiones, y para las cuales hay empresas en las CDVs agrícolas o vinculadas con ellas practicándolos. Con respecto al medio ambiente por ejemplo, la RSE implica la prevención de contaminación de diferentes maneras, p.ej. el escape de materiales tóxicos de una planta sea en el aire, agua o suelo; el uso sostenible de recursos según la frase de "reducir, reutilizar y reciclar" (Lozano, Romero, \& Serrano, 2003); la mitigación del cambio climático por la prevención de la deforestación o el cambio en el uso de combustible a uno menos contaminante (Leguizamón \& Ickis, 2005); y, la protección del hábitat y la biodiversidad por medio de, por ejemplo, actividades menos intrusivas y más respetuosas de la flora y fauna local (Ickis, Flores, \& Ickis, 2006). Sobre prácticas laborales, la RSE implica, entre otras cosas, desarrollo humano y capacitación de los trabajadores y empleados (Leguizimón \& Prado, 2006); un compromiso referente a la salud y seguridad en el trabajo; con todo en base de un diálogo y un esfuerzo por acciones concretas de mejorar las condiciones de trabajo y la protección social (Leguizamon \& Ickis, 2005). En cuanto al tema del desarrollo de la comunidad, la RSE implica que la organización demuestre un compromiso de dialogar e involucrarse con los problemas locales y la búsqueda de soluciones (González \& Márquez, 2005); crear el empleo por la oferta de puestos de trabajo; promover la generación de riqueza e ingresos por la compra de bienes y servicios de empresas locales (Fernández, Trujillo, \& Gutiérrez, 2003); apoyar el acceso a tecnología y educación en general; e invertir en infraestructura y actividades del beneficio de la gente local si trabajan directamente con la empresa o no (Lozano, Romero, \& Serrano, 2003). Hay que señalar que no todas las empresas trabajan los mismos temas o en la misma manera. No obstante, la difusión de la RSE en las CDVS agrícolas genera interés tanto sobre sus antecedentes como en su futuro potencial (Ickis, Leguizamón, Metzger, \& Flores, 2009).

El concepto de cadena de valor (CDV) también tiene largos antecedentes. Algunos autores hacen referencia a los estudios de las diferentes industrias en los EE. UU. en los años 40; otros citan los estudios sobre el flujo de materia prima de las ex colonias en África a las empresas mercantiles en los años 20 (Bolwig, Du Toit, Riisgaard, \& Halberg, 2008). En América Latina y el sector agrícola en particular, un hito importante fueron los esfuerzos por la Michigan State University (MSU) en Puerto Rico, Colombia, Brasil y Bolivia en los años 60 y 70 (Harrison, Henley, Riley, \& Shaffer, 1974). Es notable que su enfoque de "sistemas alimenticios", o sea las relaciones verticales entre diferentes actores y diferentes etapas en múltiples CDVs alimenticios, haya sido criticado por no haber tomado en cuenta desde su inicio los pequeños productores, promoviendo la reducción costos y mermas sin tomar en cuenta las implicancias para la pobreza rural (Bromley \& Symanski, 1974).

Esta tensión entre diferentes perspectivas (p.ej., campo versus ciudad), actores/promotores (p.ej., productores versus supermercados o procesadores), y objetivos (eficiencia de la empresa versus ingresos de sus proveedores) sigue vigente en muchos trabajos sobre las CDVs. Más aún, reflejan una problemática mayor del sector público en muchos países. Los recursos disponibles no son suficientes para cubrir todas las demandas de la sociedad. Entonces hay una necesidad de buscar aliados estratégicos privados para tratar de hacerlo.

Esta situación es el producto de una serie de tendencias relacionadas con la transformación del propio sector agroindustrial en América Latina que incluyeron la privatización de em- 
presas estatales y organizaciones públicas dedicadas a la distribución de alimentos (Reardon \& Timmer, 2012). Las empresas privadas: supermercados, procesadores y empresas vendedoras de insumos, lanzaron una serie de iniciativas para diversificar productos y servicios, reducir sus costos, y mejorar la calidad y de esta manera ganar espacio en el mercado frente a la competencia existente. En el caso de los supermercados, introdujeron tiendas más grandes y a la vez captaron economías de escala vía almacenes privados (en lugar de mercados mayoristas públicos); contratos formales o informales con agricultores, en base a estándares privados; $y$, hasta trabajar con proveedores particulares a veces capacitados por ellos mismos. Algunos desarrollaron relaciones comerciales simbióticas con empresas agroindustriales en ciertos rubros como productos lácteos o de carne (Farina, Gutman, Lavarello, Nunes, \& Reardon, 2005). Varias de estas prácticas innovadoras (p.ej. el uso de contratos) han sido incorporadas por procesadores de alimentos (Bollo, 2008), cadenas de comida al paso (Llaque Ramos, 2008), o empresas de agroexportación (Gómez, 2008, Leguizamon \& Ickis, 2005).

Dadas estas experiencias y estudios de las CDVs agrícolas, se puede identificar dos principales o mega enfoques: el vertical y el horizontal o territorial. En el vertical, el análisis se concentra en la relación entre diferentes etapas, procesos y actores, desde la producción hasta el consumo final por un solo producto (Ickis, Leguizamón, Metzger, \& Flores, 2009). Dentro del enfoque vertical, los temas de interés van desde los más tradicionales como: 1) la eficiencia técnica y económica - o cómo introduciendo nuevas tecnologías se puede reducir los costos y los márgenes al beneficio de los trabajadores, productores y consumidores (Escobal \& Cavero, 2012; Farina, Gutman, Lavarello, Nunes, \& Reardon, 2005); 2) infraestructura - tanto pública como privada, y 3) capacitación en múltiples dimensiones - desde el uso de tecnología disponible, a la negociación sobre los términos de compra/ venta, así como en temas de gerencia de una organización, p.ej, cooperativa, empresa particular (Fernández, Trujillo, \& Gutiérrez, 2003). Se incluye a la vez los temas como 4) la gobernanza o si la CDV es una en que los productores son líderes en el proceso de cambio o son los actores más cercanos a los usuarios finales los que tienen mayor influencia sobre qué, cómo, dónde y cuándo hay que producir lo que se produce - en este contexto, temas como estándares de calidad, certificación o registro también son importantes (Berdegué, Balsevich, Flores, \& Reardon, 2005); 5) políticas y procesos empresariales - o cómo cambiando el dónde y el quién compra los productos o la materia prima se puede mejorar los ingresos de los agricultores y la calidad para los usuarios/clientes finales (Bollo, 2008; Ickis, Leguizamón, Metzger, \& Flores, 2009); 6) políticas gubernamentales, p.ej. aranceles, impuestos, regulaciones; $y, 7$ ) en múltiples formas, el medio ambiente, o sea desde el uso de recursos naturales (renovables y no renovables), protección de la biodiversidad hasta el cambio climático (Meinzen-Dick, Deveaux, \& Antezana, 2009). Cabe señalar que dichos temas no son mutuamente exclusivos. Al contrario, parte de la dificultad y el debate en la implementación del enfoque vertical tiene que ver con dónde empezar.

Buena parte de la decisión sobre qué CDVs enfocar además de qué hacer al respecto, tiene que ver con quién es el promotor de dichas iniciativas (Bolwig, Du Toit, Riisgaard, \& Halberg, 2008). Puede ser una empresa, una organización no gubernamental (ONG), o un centro de investigación internacional (Bernet, Lara, Urday, \& Deveaux, 2002; Wheatley, Scott, Best, \& Wiersema, 1997). Típicamente, los promotores deciden los productos y lugares donde se van a trabajar y establecen los parámetros de los términos de interacción, pero ofreciendo a la vez elementos e incentivos a sus colaboradores. Dada la importancia, o la necesidad de captar los beneficios de ir a escala (Austin \& Chu, 2006) y el hecho de que el gobierno y ONGs, entre otros, pueden tener interés en ver actividades de esta naturaleza desarrollarse en otros sitios y/o sobre otros productos, hay también iniciativas relacionados con CDVs que tienen un enfoque horizontal o territorial.

El enfoque territorial se refiere a CDVs múltiples, sea por la misma materia prima (p.ej. café) o grupos de cultivos en su conjunto (cultivos Andinos), ubicadas en un territorio (región o distrito o corredor) particular (Ferris, Best, Lundy, Osterta, Gottret, \& Wandschneider, 2006; Maruyama, Alva, \& Torero, 2009). El énfasis está basado más en la oferta - que sea actual (lo que se produce en la zona) o potencial (lo que se podría producir bajo el mismo clima, suelo, mano de obra) - que en la demanda aunque en la práctica esto cambia rápidamente como parte del proceso dinámico asociado con el uso de este enfoque. En este sentido, el desarrollo de nuevos (o mejorados) productos tiene importancia como una fuente de mayores utilidades, ingresos y empleo que los costos o calidad en sí. Otros temas que se consideran bajo este enfoque incluye el medio ambiente, como la conservación de la biodiversidad, la reducción de pobreza rural o ambos (Scott, 2011).

En el ámbito empresarial, hay varias instancias en las que los agronegocios en Latinoamérica han participado en estas iniciativas territoriales con beneficios mutuos tanto para ellas nuevas fuentes de materia prima, acceso a nuevos mercados como para la sociedad - generar empleo, ingresos (Sanborn \& Delgado, 2006; Weidemann Associates, 2008). No obstante, el enfoque territorial también tiene sus desafíos. Mientras el enfoque horizontal está más abierto a múltiples cadenas a la vez, tiene que reducir el número de posibles puntos de intervención para implementar sus actividades. En términos más empresa- 
riales, implica buscar opciones que tienen mayor potencial de dar una rentabilidad sostenible para hacer sus actividades de RSE a la vez estén sostenibles en el tiempo.

En resumen, tanto el enfoque vertical que el territorial han ofrecido oportunidades para que las empresas y la sociedad obtengan beneficios. A la vez, la experiencia de las últimas décadas ha señalado también las limitaciones de ambos tipos de iniciativas. Por las intervenciones verticales, implica reconocer que no a todos los pequeños productores les interesa modificar sus hábitos de siembra y cosecha para lograr una producción agrícola más intensiva y netamente comercial (Leguizamón, Petry, \& Hernández, 2007), ni que todos ellos producen los cultivos que le interesa a una u otra empresa privada (Escobal \& Cavero, 2011). Más aún, los cambios necesarios para lograr objetivos sociales vía iniciativas involucrando a las empresas privadas, a veces depende más de decisiones que están más cerca al consumidor final que al productor (Bolwig, Du Toit, Riisgaard, \& Halberg, 2008; Ferris, Ferris, Best, Lundy, Osterta, Gottret, \& Wandschneider, 2006). Además, para lograr una sostenibilidad, las actividades de RSE dentro de las CDVs tienen que replicarse para generar los ingresos necesarios para financiarlos (Austin \& Chu, 2006). Algunas iniciativas privadas pueden lograr dicha sostenibilidad por sí solas. Pero la reciente experiencia empresarial en Latinoamérica sugiere que iniciativas privadas aisladas muchas veces no llegan a alcanzar la masa crítica para sostener sinergias retroalimentarias entre sí mismas. Se beneficiarían de un cierto apoyo gubernamental que incluye infraestructura (p.ej., carreteras) y la difusión masiva por parte de entidades públicas de las lecciones aprendidas después de la experiencia inicial tipo piloto. Al mismo tiempo, iniciativas territoriales tarde o temprano tienen que interesar a empresas privadas específicas para maximizar los potenciales beneficios en términos de generar empleo, ingresos, capacitación, acceso a mercados (Weidemann Associates, 2008) e incorporar un mecanismo de inclusión a entidades públicas para su difusión masiva y sostenibilidad en tiempo.

Por el lado metodológico, el enfoque vertical en las CDVs de agronegocios ofrece un marco conceptual manejable como una manera de impulsar nuevas iniciativas en la práctica que incorporan la RSE (Ickis, Leguizamón, Metzger, \& Flores, 2009). Pero faltan unas pautas operacionales integradas para ir a escala (Austin \& Chu, 2006). Por otro lado, el enfoque territorial ofrece una perspectiva suficientemente amplia desde el inicio. Sin embargo, las capacidades y recursos (p. ej, datos) requeridos para manejar un modelo horizontal/territorial, por lo menos a veces, son tan exigentes (Maruyama, Alva, \& Torero, 2009) que limitan, o excluyen su aplicación en una forma masiva. Además, en la medida que el enfoque horizontal dependa más de acciones gubernamentales (Austin \& Chu, 2006), el método de implementar- lo tiene que ser relativamente fácil de aplicar. De lo contrario, el tiempo necesario para identificar recomendaciones en base del enfoque dejaría poca oportunidad después para su ejecución antes de un cambio del gobierno y el proceso tendrá que empezar de nuevo desde su inicio. Todo esto sugiere la utilidad de un enfoque vertical-horizontal (Mitchell, Shepherd, \& Keane, 2011) para complementar actividades netamente privadas en el ejercicio de la RSE. Esto implica a la vez una participación por parte del sector público en decidir sobre dónde se va a trabajar. Además se sugiere la utilidad de un instrumento o serie de pautas que por lo menos en la primera instancia debe ser: 1) relativamente flexible y fácil para utilizar, pero que a la vez 2) sugiere qué hay que hacer e indique dónde y con qué producto hay que hacerlo.

\section{METODOLOGIA: HACIA LA DE PRIORIZACIÓN DE CDVS AGRICOLAS PARA LA RSE}

Se propone una matriz de factores para dar sustento a un proceso de priorización y selección de CDVs agrícolas para impulsar su desarrollo por la inclusión de la RSE. Dicha metodología debe ayudar a enfocar una cadena o grupo de cadenas y el sitio donde funciona. En el caso de CDVs agrícolas, eso implica dos componentes esenciales: 1) el producto (o productos) o la parte vertical y 2) el lugar(es) o la parte territorial, tomando en cuenta por ambos componentes la dimensión de la RSE.

Mientras se propone incorporar ambos enfoques en esta metodología, se da preferencia al inicio a la dimensión vertical como una manera de dar prioridad a la competitividad y así asegurar el interés del sector privado en el proceso y sus implicancias para las inversiones que se esperan catalizar como resultado en lugar de fijarse en una región y tratar de incentivar la participación del sector privado después. Por otro lado, se necesita una serie de preguntas o indicadores explícitos vinculados con la RSE como una manera de evaluar el potencial de las diferentes CDVs de aprovechamiento en sus respectivos agronegocios. En ese sentido, la idea es considerar la RSE como una manera de hacer negocios, y no una cuestión aparte de estos, si su práctica va a ser sostenible en tiempo. Estos indicadores sirven también para facilitar la inclusión y la promoción de dialogo entre los varios grupos interesados anticipando la importancia de su participación en la implementación de cualquier potencial iniciativa. En ese sentido, el desarrollo organizacional de apoyo a alianzas público-privadas existentes en relación a una u otra CDV tiene que figurar como una manera de evaluar el potencial institucional para ejecutar cualquier nueva iniciativa. 


\section{Criterios operacionales: el enfoque vertical}

En la práctica, la lista de criterios operacionales relacionados con el enfoque vertical (Tabla 1) incluye lo siguiente:

- Debe tener una masa crítica en términos de producción anual de, por lo menos, más de 10.000 TM/año.

- Por la misma razón, las cadenas deben haber logrado un nivel de exportaciones o ventas domésticas mínimas (p.ej., de más de 10.000 TM) con la idea de que haya un cierto volumen y valor ya establecido, como un indicador que el producto ya ha ganado cierto espacio en el mercado.

- Por las tendencias en los precios y consumo per cápita además por las características del mismo producto, la demanda está creciendo.

- Los precios nacionales son competitivos con los internacionales.

- Hay potencial para mejoras tanto técnicas (i.e., productividad) como económicas (i.e., costos por unidad producida) vía la adopción de tecnología mejorada disponible y mejoras en la infraestructura de producción y de pos cosecha.

- La cadena está dentro de los parámetros de actividades de organizaciones de innovación tecnológica nacionales.

- Mejoras tecnológicas existen. Más bien, el próximo paso sería el implementar las recomendaciones ya señaladas.

- $\quad$ El producto tiene ciertas ventajas comerciales por su sabor, consistencia frente a actuales o potenciales competidores, p. ej. sus características genéticas, época del año en que se produce,

- etc.

No se pretende que dicha lista sea definitiva, pero más bien apunta a temas que pueden sugerir indicadores adicionales del mismo enfoque (Kula, Downing, \& Field, 2006, pp.1722; Webber \& Labaste, 2010, pp.28-36; Wheatley, Scott, Best, \& Wiersema , 1997, p.41-55). Pero tampoco es útil generar una lista tan larga que se requiere demasiado tiempo en su aplicación. De todas maneras, si hay indicios de que las señales del mercado no son tan promisorias, se genera una bandera roja con respecto a dicha cadena sin importar otras consideraciones.

Al mismo tiempo los indicadores del enfoque vertical sugieren una serie de criterios adicionales relacionados con la RSE (Perú 2021, 2010) como una manera de extender su interpretación en el ejercicio de priorizar las CDVs. Ellos incluyen:

- Existe potencial de que los beneficios puedan llegar a un grupo significativo de unidades agrícolas; que no sea un cultivo en el que la producción está concentrada en relativamente pocas unidades.

- Se favorece un cultivo producido a escalas que ofrecen mayor potencial para ayudar a reducir la pobreza y la pobreza extrema rural.

- Hay potencial con las cadenas seleccionadas para no solamente mejorar ingresos, sino también para tener un impacto ambiental positivo.

- Existe la posibilidad de que algunas de las actividades ambientales vinculadas a las cadenas seleccionadas puedan ser remuneradas (p.ej. por bonos de carbono), y de esta manera integrar las actividades de las cadenas con el cuidado del medio ambiente.

- Las tecnologías innovadoras están aptas también para productores pequeños.

- La cadena incluye nichos o segmentos que favorecen pequeños productores y así ofrece la potencial de mejorar el acceso a la tecnología.

- Las etapas en la cadena ofrece también oportunidades de generar empleo.

\section{Criterios operacionales: el enfoque territorial}

Además de una variación entre tipos de productos o cultivos en sí, hay que reconocer que una CDV tiene una dimensión territorial. En ese sentido, un país puede caracterizarse por marcadas diferencias entre las regiones en términos de sus climas, suelos, alturas entre otras características en las cuales las CDVs donde y pueden operar. Los indicadores operacionales para esta dimensión incluyen (Tabla 2):

- El territorio tiene una masa crítica de producción.

- Tiene infraestructura, p.ej. carreteras que dan acceso al mercado.

- Ciertas características como clima, altura etc. que le dan una ventaja competitiva por la

- $\quad$ época del año en que se puede cosechar o la calidad del producto que sale al mercado.

- Es una zona de biodiversidad que se puede proteger.

- Hay ciertos servicios relacionados con la producción y venta de la cadena que están disponibles.

- Ya hay ciertas empresas, organizaciones trabajando en la CDV en la zona.

- Se puede de expandir el área bajo cultivo en la zona.

- Hay tecnologías disponibles para mejorar la productividad y/o calidad del producto en

- dicha zona.

- Mejor infraestructura podría tener un impacto significativo. 
TABLA 1. Enfoque vertical: Pautas para la priorización de Cadenas de Valor

\begin{tabular}{|c|c|c|c|}
\hline Enfoque/ indicador & $\begin{array}{l}\text { Cadena/cultivo } \\
1234\end{array}$ & & $\begin{array}{l}\text { Cadena/cultivo } \\
1234\end{array}$ \\
\hline Vertical (Oferta y demanda) & & RSE/ indicador & \\
\hline Ventas anuales (Mercado doméstico/exportación (> 10.000 MT) & & Muchos productores venden poco & \\
\hline Se produce en una sola región, o varias & & $\begin{array}{l}\text { Incrementa la posibilidad que se produzca en } \\
\text { una zona de pobreza }\end{array}$ & \\
\hline Rendimientos (Promedio MT/ha versus potencial) & & $\begin{array}{l}\text { Rendimientos mejorados son alcanzables por } \\
\text { todo tamaño de productor }\end{array}$ & \\
\hline Costos por kg producido (Promedio versus potencial) & & $\begin{array}{l}\text { Hay economías de escala a un modesto nivel } \\
\text { de producción }\end{array}$ & \\
\hline El cultivo/producto es diferenciado o genérico & & Su cultivo ayuda a mantener la biodiversidad & \\
\hline Hay nuevas tecnologías desarrolladas y disponibles & & $\begin{array}{l}\text { Dichas tecnologías están aptas para diferentes } \\
\text { tamaños de productores }\end{array}$ & \\
\hline Su producción y venta esta mecanizada & & O genera mucho empleo & \\
\hline $\begin{array}{l}\text { Que tan competitiva es la cadena frente a otras regiones, nacionales, } \\
\text { internacionales }\end{array}$ & & $\begin{array}{l}\text { Hay nichos o segmentos para pequeños } \\
\text { productores y/o nuevas empresas }\end{array}$ & \\
\hline
\end{tabular}

Fuente: Desarrollado para este estudio en base de Kula, Downing, \& Field (2006); Webber \& Labaste (2010), Wheatley; Scott, Best, \& Wiersema (1997) y consultorías previas.

\section{TABLA 2. Enfoque territorial: Pautas para la priorización de Cadenas de Valor}

\begin{tabular}{lll}
\hline Enfoque/ indicador & Cadena/cultivo & Cadena/cultivo \\
& 12234 & $\mathbf{1} 24$ \\
\hline Territorial (Ubicación/dominio) & & RSE/ indicador \\
\hline
\end{tabular}

La cadena está ubicada en una zona/territorio que tiene una masa crítica de producción

Hay un potencial para expandir el área sembrada

Tiene infraestructura, p.j. carreteras, puertos, ferrocarriles que dan acceso al mercado

Tiene ciertas características (altura, clima suelo que da una ventaja competitiva)

Hay mayor potencial con mejoras en infraestructura

Hay tecnologías y otros servicios disponibles para la zona

Hay incentivos económicos para proteger el medio ambiente de la zona

Los incentivos están asociados con la producción del cultivo de la cadena

Hay empresas privadas ubicadas en la zona trabajando ya con el cultivo

Hay organizaciones de apoyo, público y privado funcionado en la zona: bancos, extensionistas vendedores de insumos
Hay una alta incidencia de pequeños productores

Hay una alta incidencia de pobreza

Dicha infraestructura esta donde están también los pequeños productores

Es una zona de biodiversidad

Estas mejoras pueden beneficiar muchos productores, personas

Están alcanzables por todos los tipos de productores

Estos incentivos son alcanzables por todo tamaño de productor

Dichos ingresos pueden mejorar los ingresos de los pequeños productores

Dichas empresas incluyen las que trabajan con pequeños productores

Hay organizaciones de apoyo que trabajan con pequeños productores

Fuente: Desarrollado para este estudio en base de Ferris, Best, Lundy, Osterta, Gottret, \& Wandschneider (2006); Wheatley, Scott, Best, \& Wiersema (1997) y consultorías previas. 
Al mismo tiempo, hay indicadores de la RSE que complementan los de agro-ecología, logística o tecnología del enfoque territorial (Tabla 2). Ellos hacen referencia a temas relacionados con la reducción de pobreza, generación del empleo, etc. e incluyen:

- Es una zona que contiene una concentración de pequeños productores.

- Hay alta incidencia de pobreza.

- Hay una alta incidencia de biodiversidad.

- Hay empresas, organizaciones ya trabajando en la zona con pequeños productores.

- Hay tecnologías mejoradas aptas para la zona y que estén disponibles y aptas para pequeños

- productores.

Hay que recordar, como en el caso de los otros indicadores, que no se pretende presentar una lista definitiva, dadas las diferentes circunstancias que podrían existir entre países. Más bien, la combinación de criterios verticales y territoriales combinados con los de RSE en conjunto ofrece una base más amplia para la selección o priorización de las CDVs.

\section{UNO EJEMPLO DE UNA CADENA Y REGIONES SELECCIONADAS}

Dada la proliferación de información tanto de estadísticas como de estudios, y la revolución informática en América Latina que ofrece acceso a ambos, es mucho más fácil hoy en día llevar a cabo una revisión de los datos secundarios de diferentes ministerios públicos, una revisión de la literatura tanto publicada como no publicada (Scott, 1995), y una serie de entrevistas informales con representantes de varios gremios, centros de investigación, especialistas en diferentes cultivos o sectores, además de funcionarios en diferentes Ministerios con el propósito de coordinación y validación de los acontecimientos.

Los resultados preliminares en el caso del Perú, señalaron que-tomando el sub-sector de los cultivos (versus productos animales) como un ejemplo, hay más de 130 potenciales candidatos para un programa de desarrollo de CDVs involucrando organizaciones públicas y empresas privadas en el país (Tabla 3). En efecto, al inicio del proceso, todos los cultivos entran como candidatos potenciales para desarrollarse más en una nueva iniciativa dirigidas a las CDVs. Por otro lado, el Perú está conformado por más de 20 regiones políticas cada una con sus sub-regiones. Dado el alto número de candidatos en términos de cultivos (139) y territorios combinados con el hecho de que los recursos son escasos, se refuerza la necesidad de establecer unos criterios de priorización. Se debe señalar, además, que si bien es importante estar informado de los últimos acon- tecimientos sobre diferentes productos y sus perspectivas comerciales incluyendo el corto plazo, los objetivos de este ejercicio están orientados más a mediano y largo plazo.

Tomando en cuenta los varios factores mencionados arriba en el caso del Perú, se logró identificar y reclasificar más de 50 cultivos (o desagregar las cifras de algunos cultivos como, por ejemplo, frijol) adicionales por criterios comerciales (Tabla 4). En principio, cualquiera de ellos podría estar seleccionado. La realidad también es que cada uno podría mejorarse de una manera u otra. No obstante, lo que se intenta es seleccionar a aquellos que tienen mayor potencial para cumplir con todos los criterios identificados.

Una calificación de las regiones en base a un índice de competitividad regional Índice de Competitividad Regional [ICR] es una manera de cuantificar las capacidades distintas entre las regiones (Tabla 5). Los resultados de este índice ofrecen un indicador de la capacidad de una región para aprovechar nuevas inversiones, realizado con la intención de que el territorio se vuelva más competitivo a nivel internacional en el futuro.

Mientras el ICR indica la competitividad actual según un conjunto de criterios, la información disponible sobre el cuadro de fondos de empresas de recursos naturales no-renovables, ofrece un indicador más específico de la capacidad financiera de los gobiernos regionales para incurrir en futuras inversiones. Hay marcadas diferencias entre regiones en términos de cantidad de recursos disponibles de dichas fuentes (Tabla 5). Ello implica la capacidad de compartir los costos asociados con el desarrollo de una cadena en términos de costos de instalar infraestructura: carreteras, mercados, facilidades portuarias, también son distintas.

\section{Cafés diferenciados: Cusco y Cajamarca}

El café es el primer producto de agroexportación peruana. Más aún mientras las exportaciones totales de café pasaron de US\$ 223 a US\$ 303 millones entre 2000 y 2005 , las exportaciones de cafés especiales pasaron de US\$13.5 millones a US\$ 69.9 millones durante el mismo periodo (Remy \& Glave, 2007). Cafés especiales incluyen al café orgánico, comercio justo, y gourmet/Premium, de las cuales el café orgánico representa alrededor del 50\%. Estas ventas han ayudado a las cooperativas a invertir en infraestructura y a facilitar que los productores tengan el incentivo y mejor capacidad de producir y vender café de la más alta calidad que ha ganado concursos internacionales en repetidas ocasiones (Junta Nacional de Café [Jnc], 2009). En otras palabras, se ha logrado un nivel de exportacion con el café diferenciado impresionante, que sirve de base para un futuro desarrollo. 
TABLA 3. Mapeo de los cultivos ( $n=139)$ en el Perú por producción anual, cerca 2007 y 2008

\begin{tabular}{|c|c|c|c|c|c|c|c|c|c|c|}
\hline \multicolumn{11}{|c|}{ Producción (ooo TM/año) de cultivo } \\
\hline Categoría & $>1.000$ & $\begin{array}{l}<1.000 \\
y>500\end{array}$ & $\begin{array}{l}>500 y \\
>150\end{array}$ & $\begin{array}{c}\langle 150 y \\
>50\end{array}$ & $\langle 50$ y $>10$ & $\langle 10 y\rangle 5$ & $<5$ & \multicolumn{3}{|c|}{ Sin información } \\
\hline $\begin{array}{l}\text { Cereales } \\
\text { raíces y } \\
\text { tubérculos } \\
\text { frijol, } \\
\text { hortalizas } \\
\text { especies }\end{array}$ & $\begin{array}{l}\text { arroz cáscara } \\
\text { papa } \\
M A D^{1} \\
\text { yuca }\end{array}$ & cebolla & $\begin{array}{l}\text { camote } \\
\text { cebada } \\
\text { grano } \\
\text { espárrago } \\
\text { olluco² } \\
\text { maíz } \\
\text { amiláceo } \\
\text { maíz choclo } \\
\text { palta² } \\
\text { trigo } \\
\text { zanahoria } \\
\text { zapallo }\end{array}$ & $\begin{array}{l}\begin{array}{l}\text { aceituna } \\
\text { (olivo) }\end{array} \\
\text { ajo } \\
\text { alcachofa } \\
\text { arveja grano } \\
\text { verde }{ }^{2} \\
\text { frijol grano } \\
\text { seco } \\
\text { haba grano } \\
\text { verde }{ }^{2} \\
\text { oca }{ }^{2} \\
\text { páprika }{ }^{3} \\
\text { pimiento }\end{array}$ & $\begin{array}{l}\text { ají } \\
\text { apio } \\
\text { arveja grano } \\
\text { seco } \\
\text { betarraga } \\
\text { brócoli } \\
\text { col } \\
\text { coliflor } \\
\text { frijol castilla² } \\
\text { lechuga } \\
\text { rabanito } \\
\text { vainita }\end{array}$ & $\begin{array}{l}\text { maca } \\
\text { mashua } 2 \\
\text { nabo } \\
\text { pallar grano } \\
\text { seco }^{2} \\
\text { palmito- } \\
\text { pijuayo } \\
\text { pepino } \\
\text { poro } 4 \\
\text { quinua }\end{array}$ & $\begin{array}{l}\text { cañihua² }^{2} \\
\text { orégano } \\
\text { tarhui }\end{array}$ & $\begin{array}{l}\text { achiote }^{2} \\
\text { acelga } \\
\text { caigua } \\
\text { frijol de palo } \\
\text { frijol loctao }{ }^{2 *} \\
\text { garbanzo } \\
\text { kiwicha }\end{array}$ & $\begin{array}{l}\text { lenteja } \\
\text { sorgo2* } \\
\text { soya } \\
\text { yacon }^{2^{*}}\end{array}$ & $\begin{array}{l}\text { albahaca } \\
\text { calabaza } \\
\text { culantro } \\
\text { espinaca }\end{array}$ \\
\hline $\begin{array}{l}\text { Frutas, } \\
\text { nueces }\end{array}$ & plátano & & $\begin{array}{l}\text { limón } \\
\text { mandarina } \\
\text { mango } \\
\text { naranja } \\
\text { papaya } \\
\text { piña } \\
\text { tomate } \\
\text { uva }^{2}\end{array}$ & $\begin{array}{l}\text { manzana } \\
\text { sandía }{ }^{4} \\
\text { tangelo } \\
\text { tuna }\end{array}$ & $\begin{array}{l}\text { aguaje } \\
\text { camu camu } \\
\text { coco } \\
\text { chirimoya } \\
\text { durazno } \\
\text { fresa } \\
\text { granadilla } \\
\text { maracuyá }\end{array}$ & $\begin{array}{l}\text { lúcuma } \\
\text { melocotón } \\
\text { melón } \\
\text { zarandaja² }\end{array}$ & $\begin{array}{l}\text { algarrobo } \\
\text { caimito } \\
\text { cocona } \\
\text { maní } \\
\text { pera } \\
\text { taperiba }\end{array}$ & $\begin{array}{l}\text { aguaymanto } \\
\text { amona } \\
\text { carambola } \\
\text { cereza* } \\
\text { damasco* } \\
\text { dátil* } \\
\text { granado* } \\
\text { guanábana } \\
\text { guayaba } \\
\text { guinda* } \\
\text { higo } \\
\text { limón dulce }\end{array}$ & $\begin{array}{l}\text { nuez del } \\
\text { Brasil } \\
\text { níspero } \\
\text { pecana } \\
\text { sauco* } \\
\text { tamarindo* } \\
\text { toronja } \\
\text { tumbo } \\
\text { zapote } \\
\text { mamey } \\
\text { marañón } \\
\text { membrillo }\end{array}$ & $\begin{array}{l}\text { arándano } \\
\text { árbol de pan } \\
\text { castaña } \\
\text { ciruela } \\
\text { humari } \\
\text { lima } \\
\text { pacae } \\
\text { pomarrosa }\end{array}$ \\
\hline Industriales & $\begin{array}{l}\text { caña de } \\
\text { azúcar }\end{array}$ & & café & $\begin{array}{l}\text { algodón } \\
\text { rama }\end{array}$ & cacao & & barbasco & anís* & & \\
\hline $\begin{array}{l}\text { plantas } \\
\text { medicinales }\end{array}$ & & & $\begin{array}{l}\text { palma } \\
\text { aceitera }^{2}\end{array}$ & & $\begin{array}{l}\text { cochinilla } \\
\text { tara }\end{array}$ & & $\begin{array}{l}\text { marigold }^{2} \\
\text { molle }^{5} \\
\text { sangre de } \\
\text { grado }^{6}\end{array}$ & $\begin{array}{l}\text { palillo } \\
\text { uña de gato* } \\
\text { té }\end{array}$ & & \\
\hline
\end{tabular}

*Hay cifras, pero no están disponibles fácilmente.

N.B La letra de imprenta indica que los datos al nivel de la región están disponibles en la página web del Minag, la letra cursiva indica que hay estimaciones de los costos de producción disponible por parte del Minag.

${ }^{1}$ Maíz amarillo duro. ${ }^{2}$ Producción 2008-10. 3Producción 2008-09. 4Producción 2004-06. ${ }^{5}$ Producción de semillas, uso mixto como producto industrial y alimentaria. ${ }^{6}$ Litros de látex. ${ }^{\top}$ Menos de $1.000 \mathrm{MT}$.

Fuente: Datos para los cultivos son del Minag citado en Perú Acorde (2009) salvo para los de 2008-10 que son de la página web del Minag y los de páprika que son de Maximixe (2010). 


\section{TABLA 4. Grupos de cultivos según el segmento que se apuntan y sus características}

\begin{tabular}{l|l|l|l}
\multicolumn{5}{l}{ Sub-sector Exportación diferenciadas: Exótico, orgánico } & \multicolumn{1}{l}{ Otro } \\
\hline Frutas exóticas & Cultivos Andinos & Cultivos industriales & Palmito-pijuayo \\
\hline Aguaje & Maca & Cacao & \\
\hline Banana orgánica & Maíz amiláceo & Café & \\
\hline Chirimoya & Maíz choclo & Cochinilla & \\
\hline Granadilla & Mashua & Tara & \\
\hline Lúcuma & Oca & & \\
\hline Pacae & Olluco & & \\
\hline Tuna & Quinoa & & \\
\hline Zarandaja & & & \\
\hline
\end{tabular}

Sub-sector Exportación no tradicional: Contraestación, calidad y costo

\begin{tabular}{|c|c|c|c|c|c|}
\hline \multicolumn{3}{|c|}{ Frutas y nueces } & \multicolumn{2}{|c|}{ Hortalizas y especies } & \multirow{2}{*}{$\begin{array}{l}\text { Menestras } \\
\text { Arveja grano seco }\end{array}$} \\
\hline Cítricos & Tropicales & Templado & Exportación & En despegue & \\
\hline Limón & Coco & Durazno & Aceitunas & Apio & Arveja grano verde \\
\hline Mandarina & Mango & Fresa & Alcachofa & Betarraga & Frijol grano seco \\
\hline Naranja & Maracuyá & Manzana & Ají & Brócoli & Frijol castilla \\
\hline \multirow[t]{10}{*}{ Tangelo } & Papaya & Melocotón & Ajo & Coliflor & Haba grano seco \\
\hline & Piña & Melón & Espárrago & Camote & Haba grano verde \\
\hline & & Pepino & Palta & Coliflor & Pallar grano seco \\
\hline & & Sandía & Páprika & Nabo & \\
\hline & & Uva & & Lechuga & \\
\hline & & & & Pimiento & \\
\hline & & & & Poro & \\
\hline & & & & Rabanito & \\
\hline & & & & Tomate & \\
\hline & & & & Vainita & \\
\hline
\end{tabular}

\section{Sub-sector Tradicional: Calidad y costo}

\section{Cultivos industriales}

Algodón

Cacao genérico

Café genérico

\section{Sub-sector Tradicional: Calidad y costo}

\section{Cultivos industriales}

Algodón

Cacao genérico

Café genérico

Fuente: Datos de Tabla 1 re-organizados para este estudio. 
TABLA 5. Competitividad, canon y pobreza por región, 2009-2010

\begin{tabular}{|c|c|c|c|c|c|c|c|}
\hline $\begin{array}{c}\text { Regiones/ } \\
\text { Departamentos }\end{array}$ & \multicolumn{3}{|c|}{2010} & \multicolumn{2}{|c|}{2009} & $\begin{array}{c}\text { Pobreza } \\
\text { (\% población) }\end{array}$ & $\begin{array}{l}\text { Extrema pobreza } \\
\text { (\% población) }\end{array}$ \\
\hline Amazonas & 24,42 & 113.136 & 5.409 & 2.793 .661 & 2.912 .206 & 59,8 & 22,1 \\
\hline Arequipa & 45,32 & $355 \cdot 039 \cdot 521$ & 48.429 .247 & 16.819 .276 & 420.288 .044 & 21,0 & 4,3 \\
\hline Ayacucho & 23,54 & 34.626 .619 & 14.132 .886 & $7.759 \cdot 312$ & 56.518 .817 & 62,6 & 30,7 \\
\hline Cajamarca & 23,17 & 416.618 .346 & 57.549 .619 & 14.955 .246 & 489.123 .210 & 56,0 & 23,1 \\
\hline Huánuco & 25,70 & 1.680 .584 & 36.021 .967 & 1.455 .739 & 4.577 .548 & 64,5 & 29,9 \\
\hline Ica & 44,49 & $69 \cdot 390.721$ & 41.957 .065 & 3.905 .555 & 86.006 .600 & 13,7 & 0,6 \\
\hline Junín & 31,66 & 64.085 .388 & 25.953 & 5.978 .591 & 106.085 .946 & 34,3 & 15,1 \\
\hline La Libertad & 42,63 & $427 \cdot 390.779$ & 36.323 .231 & 11.168 .979 & 480.516 .823 & 38,9 & 15,4 \\
\hline Lambayeque & 41,40 & 118.329 & 0 & 1.486 .431 & 1.630 .713 & 31,8 & 4,9 \\
\hline Lima/1 & 73,57 & 73.867 .115 & 0 & 9.268 .544 & $119 \cdot 437.046$ & 15,3 & 1,2 \\
\hline Loreto & 32,99 & 0 & 95.347 .175 & 97.319 & $97 \cdot 319$ & 56,0 & 21,2 \\
\hline San Martín & 25,18 & 444.987 & 0 & 922.555 & 1.387 .893 & 44,1 & 9,2 \\
\hline Tacna & 41,27 & 230.559 .686 & 0 & 4.777 .622 & 311.655 .818 & 17,5 & 2,1 \\
\hline Tumbes & 35,46 & 0 & 0 & 18.094 & 18.094 & 22,1 & 2,2 \\
\hline Ucayali & 27,16 & 0 & 0 & 43.727 & 43.727 & 29,7 & 10,4 \\
\hline TOTAL & & $3,243,359,011$ & $578,741,951$ & $139,390,213$ & $3,961,491,175$ & & \\
\hline
\end{tabular}

*Nuevos soles.

LIMA $/{ }^{1}$ incluye el Callao.

Fuente: ICR: Centro de Negocios de la Pontificia Universidad Católica del Perú [Centrum], 2010; Canon, regalías y el derecho de vigencia de consultas con funcionarios del Ministerio de Agricultura [Minag] sobre datos de Ministerio de Energía [MEM] y Minas, del Instituto Nacional de Estadística e Información [INEI] y del Encuesta Nacionales de Hogares [ENAHO] 2006-2009; Pobreza y pobreza extrema Centro Nacional de Planeamiento Estratégico [Ceplan] (2010).

Estos éxitos han generado también una serie de nuevos desafios que incluyen: 1) conseguir títulos y registrar las tierras de los pequeños agricultores cafetaleros (La Serna, 2010); 2) renovar las plantaciones, que en promedio tienen 20 años de explotación cuando una vida rentable es de 10 años, que concretamente implica renovar un estimado de 250.000 ha en los proximos años (Inc, 2009); 3) reforzar los esfuerzos para que más productores opten por la asociatividad y aprovechen de las economías de escala asociada con ella (Minag, 2002); 4) eliminar el doble impuesto sobre ventas de los pequeños agricultores-una vez por la entrega a la coo- 
perativa y otra vez por la venta del café en el mercado, y exonerar tributos para los ingresos anuales de las personas naturales, generado por la actividad agraria, que no excedan 20 unidades impositivas tributarias (De Week \& La Torre, 2007); 5) evaluar e introducir variedades mejores de café - mejorando los procesos de control fitosanitario sobre el material de otros países que ha perjudicado el proceso últimamente; 6) mejorar los procedimientos de pos-cosecha para garantizar la calidad de la materia prima y no contaminar el medio ambiente en el proceso; 7) implementar una manera de registrar las exportaciones de cafés diferenciados por parte de ADUANAS como un dato separado de las cifras de las exportaciones de café genérico (Remy \& Glave, 2007); y, 8) evaluar en qué medida los programas para la venta de bonos del carbono para el cuidado del medio ambiente en la reforestación de las parcelas de café podrían servir como una fuente de ingreso significativo adicional y a la vez ayudar en la financiación del mismo. En otras palabras, ya existe una agenda de trabajo técnico y organizacional, que no requiere estudios adicionales para precisar lo que hay que hacer. Más bien se puede enfocar la implementación de las recomendaciones.

Según la Jnc, 2009, 85\% de la producción del café está cosechada en cinco regiones: Amazonas, Cajamarca, Cusco, Junín y Puno. Más aún, en cada uno de esas regiones existe un porcentaje significativo de pobreza rural y pobreza extrema (Tabla 5). Por otro lado, los gobiernos regionales como los de Cajamarca y Cusco tienen fondos de canon minero, sugiriendo por lo menos la posibilidad de promover la idea de una alianza estratégica entre el gobierno nacional y el gobierno regional para el beneficio de las personas trabajando en las cadenas de café diferenciado, la región y el país entero.

\section{Observaciones finales}

La privatización y liberalización de los mercados domésticos e implementación de los Tratados de Libre Comercio en Latinoamérica han contribuido a la generación de lo que algunos observadores caracterizan como una nueva relación entre la empresa y la sociedad en América Latina. Dicha relación implica involucrar a las empresas en actividades de la RSE. Como parte de la evolución de la relación empresa-sociedad se incluye un mayor interés por parte de gobiernos y del sector privado en lograr alianzas estratégicas con el propósito de hacer más de lo que uno podría hacer por sí solo y así fomentar la RSE en el espacio que representa las CDVs. En este sentido, se ha presentado una lista de factores a tomar en cuenta para priorizar dichas iniciativas en el sector de agronegocios como un primer paso para catalizar una más eficiente y eficaz alianza pública-privada en facilitar la RSE en las CDVs en el sector agrícola en Lati- noamérica. Se entiende a la vez, que los resultados de la evaluación de los factores es nada más que el primer paso en un proceso dinámico involucrando una serie de actores, pero en la medida en que las CDVs seleccionadas sean más promisorias la probabilidad de éxito es más segura tanto para el sector privado como el público. Los temas para futura investigación incluyen la posible asignación de pesos a los diferentes factores, además de la modificación de la matriz en sí, en base de nuevos acontecimientos en diferentes países. Además sería interesante desarrollar otras matrices para otros sectores como la de construcción (inmobiliaria, carreteras) o confecciones de ropa, solamente por mencionar un par de ejemplos.

\section{REFERENCIAS}

Anderson, K., \& Valenzuela, E. (2010). América Latina: ¿Cómo repercuten en los mercados y el bienestar las reformas de la política agraria y del comercio? Revista CEPAL, (100), 147-168.

Austin, J. E., \& Chu, M. (2006). Business and low-income sectors: finding a new weapon to attack poverty. ReVista - Harvard Review of Latin America, 6 (1), 3-5.

Berdegué, J. A., Balsevich, F., Flores, L., \& Reardon, T. (2005). Central American supermarkets' private standards of quality and safety in procurement of fresh fruits and vegetables. Food Policy, 30 (3) 254-269.

Bernet, T., Lara, M., Urday, P., \& Deveaux, A. (2002). El reto de vincular a los pequeños productores de papa con la agroindustria. Revista Latinoamericana de la Papa, 13 (1), 1-23.

Bollo, G. (2008, Mayo). Negocio de hojuela y bocaditos a base de papas nativas. Primer congreso nacional de la papa. Huancayo, Peru, 1. Consultado en 18 Febrero, 2013, de www.minag.gob.pe,.

Bolwig, S., Du Toit, A., Riisgaard, L., \& Halberg, N. (2008). Integrating poverty, gender and environmental concerns into value chain analysis. A conceptual framework and lessons for action research. [Working paper no 2008/16]. Danish Institute for International Studies, Copenhagen, DK.

Bromley, R. J., \& Symanski, O. R. (1974). Marketplace trade in Latin America. Latin American Research Review, 9 (3), 3-38.

Centro de Negocios de la Pontificia Universidad Católica del Perú. (2010). Índice de competitividad regional del Perú. Consultado en 18 Febrero 2013, de http://www.centrum.pucp.edu.pe/es/contenido.php?834/ investigacion_publicacion/icrp_2010_ndice_competitividad_regional_ del_peru.html\&idp=309.

Centro Nacional de Planeamiento Estratégico. (2010). Síntesis regional. Recursos, potencialidades y crecimiento. Consultado en 18 Febrero 2013, de http://www.ceplan.gob.pe/documents/10157/205fd1b4-a31C4993-a8dc-17a3516c2875.

D’Alessio, F., \& Marquina, P. (s/f). Historia y evolución de la responsabilidad social empresarial. Manuscrito sin publicar. Lima: CENTRUM Católica Graduate Business School.

De Week, C., \& La Torre, C. (2007). Un futuro para el café pasado. Perú Hoy (Dic.).

Drost, S., Van Wijk, J., \& Vellema, S. (2011). Development value chains meet business supply chains: the concept of global value chains unraveled. [Working paper no 2011/8]. Maastricht School of Management, Maastricht, Holanda, NL. 
Escobal, J., \& Cavero, D. (2012). Transaction costs, institutional arrangements and inequality outcomes: potato marketing by small producers in rural Peru. World Development, 40 (2), 329-341.

Espinal, C. F., \& Martínez, H. (2006). Agroindustria y competitividad en Colombia. Estructura y dinámica 1992-2005. Instituto Interamericano para la Cooperación Agrícola, Bogotá, CO.

Farina, E. M. M. Q., Gutman, G., Lavarello, P.L., Nunes R, \& Reardon, T. (2005). Private and public milk standards in Argentina and Brazil. Food Policy, 30 (3), 302-315.

Fernández, D. V., Trujillo, D. V., \& Gutiérrez, R. (2003). Induplama y las cooperativas de trabajo asociado, 1991-2002 (B). [SEKN Case No. SKS022]. Harvard Business School Publishing. Boston, MA, USA.

Ferris, S., Best, R., Lundy, M., Ostertag, C., Gottret, M., \& Wandschneider, T. (2006). Strategic paper: a participatory and area-based approach to enterprise development. Centro Internacional de Agricultura Tropical. Cali, COL.

Flores, J., Ogliastri, E., Peinado-Vara, E., \& Petry, I. (2007). (Eds) El argumento empresarial de la RSE: 9 casos de América Latina y el Caribe. Washington: Banco Interamericano de Desarrollo.

Gómez, R. (2008). Agricultura comercial moderna en el Perú: el caso de la agricultura de exportacion no tradicional (1995-2007). En: Damonte, G., Fulcrand, B., \& Gómez, R. (Eds). Seminario Permanente de Investigacion Agraria. XII: Perú: el problema agrario en debate. Lima: SEPIA.

González, R. A., \& Márquez, P. (2005). Las iniciativas sociales de Ron Santa Teresa. (SEKN Case No. SKS-049). Harvard Business School Publishing. Boston, MA, USA.

Harrison, K., Henley, D., Riley, H., \& Shaffer J. (1987). Improving food marketing systems in developing countries: experiences from Latin America. (Research report no. 6), Michigan, Latin American Studies Center, East Lansing, Michigan State University.

Ickis, J, Flores, J, \& Ickis, C. (2006). The magic of butterflies: ecological development in Costa Rica. ReVista. Harvard Review of Latin America, $6(1), 10-12$.

Ickis, J, Leguizamón, F, Metzger, M, \& Flores, J. (2009). La agroindustria: campo fértil para los negocios inclusivos. Academia, Revista Latinoamericana de Administración, 43, 107-124.

Junta Nacional de Café. (2009). Directorio cafetalero 2010. Lima: Jnc.

Kula, O., Downing, J., \& Field, M. (2006). Globalization and the small firm: a value chain approch to economic and poverty reduction. (Micro report No. 42), Washington, D.C, United States Agency for International Development.

La Serna, J. C. (2010). De golondrinos a enganches a empresarios exportadores. Historia del café y el cooperativismo cafetalero en la selva central. Lima: Jnc.

Leguizimón, F, \& Ickis, J. (2005). Pantaleón. (SEKN Case No. SKS-059). Harvard Business School Publishing. Boston, MA, USA.

Leguizimón, F, \& Prado, A. (2006). Grupo CSU-CCA. (SEKN Case No. SKS076). Harvard Business School Publishing. Boston, MA, USA.

Leguizamón, F., Petry, I., \& Hernández, M. (2007). La RSE y los negocios con los sectores de bajos ingresos: los casos de PALI y Tierra Fértil. En: Flores, J., Ogliastri, E., Peinado-Vara, E., \& Petry, I. (Eds). El argumento empresarial de la RSE: 9 casos de América Latina y el Caribe. Washington, D.C: BID (Banco Interamericano de Desarrollo.

Llaque Ramos, L. J. (2008). Ciclo de vida e innovación de la industria avícola peruana 1970-2006. Anales científicos de la UNALM (Universidad Nacional Agraria La Molina), 69 (2), 9-18. Disponible en: http://www. lamolina.edu.pe/Investigacion/web/anales/2008/2.pdf.
Lozano, G, Romero, C, \& Serrano, L. (2003). Supermercadosinternacionales HEB y el Banco de Alimentos de Cáritas de Monterrey, A. C. SEKN Case No. SKS-003. Boston, MA: Harvard Business School Publishing.

Maruyama, E, Alva, C, \& Torero, M. (2009). Análisis de cuellos de botella y priorización de inversiones para el desarrollo de la Sierra Rural del Perú (Versión preliminar). Washington, D.C.: IFPRI (International Food Policy Research Institute).

Maximixe. (2010). Riesgos de mercado (Agosto): paprika. Informe preparado para el Ministerio de Agricultura. Lima: Maximixe.

Meinzen-Dick, R, Devaux, A, \& Antezana, I. (2009). Underground assets: potato diversity to improve the livelihoods of the poor. International Journal of Agricultural Sustainability, 7 (4), 235-248.

Minag (Ministerio de Agricultura). (2002). Plan estratégico de la cadena productiva del café. Lima: Minag.

Mitchell, J, Shepherd, A, \& Keane, J. (2011). An introduction. En: Mitchell, J, Coles, C. (Eds) Markets and rural poverty. Upgrading in value chains. Ottawa: IDRC (International Development Research Centre).

Perú 2021. (2010). Indicadores Ethos- Perú 2021 de responsabilidad social empresarial. Lima: Perú 2021.

Perú Acorde. (2009). Estudio económico productivo del Perú. 4. ed. Lima: Perú Acorde.

Piñones Vázquez, S, Acosta Avila, L. A, \& Tartanac, F. (2006). Alianzas productivas en agro-cadenas. Experiencias de la FAO en América Latina. Santiago: FAO.

Reardon, T, \& Timmer, C. P. (2012). The economics of the food system revolution. Annual Review of Resource Economics, 4, 225-264.

Reficco, E, \& Ogliastri, E. (2009). Empresa y sociedad en América Latina: una introducción. Academia, Revista Latinoamericana de Administración, 43, 1-25.

Remy, M, \& Glave, M. (2007). Cafetaleros empresarios. Lima: Oxfam-IEP (Instituto de Estudios Peruanos).

Sanborn, C, \& Delgado, A. J. (2006). Palmas del espino: harvesting hope in the Upper Huallaga. ReVista. Harvard Review of Latin America, 6 (1), 6-9.

Scott, G. (1995). Wall-to-wall fieldwork: secondary data collection for food systems research. En: Scott, G. (Ed) Prices, products, and people. Analyzing agricultural markets in developing countries. Boulder: Lynne Rienner.

Scott, G. (2011). Plants, people, and the conservation of biodiversity of potatoes in Peru. Natureza \& Conservação, 9 (1), 21-38.

Tromben, C. (2011). Capitalismo 3.o. AMÉRICAECONOMÍA PERÚ, Febrero.

Wced (World Commission on Environment and Development). (1987). Our Common Future. Oxford: Oxford University Press.

Webber, C. M, \& Labaste, P. (2010). Building competitiveness in Africa's agriculture. Washington, D.C: International Bank for Reconstruction Development.

Weidemann Associates. (2008). Final evaluation of USAID/Peru poverty reduction and alleviation (PRA) activity. Washington, D.C: Weidemann Associates.

Wheatley, C, Scott, G, Best, R, \& Wiersema, S. (1997). Métodos para agregar valor a raíces y tubérculos: manual para el desarrollo de productos. Cali: CIAT (Centro Internacional de Agricultura Tropical).

Wilkinson, J, \& Rocha. R. (2009). Agro-industry trends, patterns and development impacts. En: da Silva, C, Baker, D, Shepherd, A, Jenane, C, \& Miranda-da-Cruz, S. (Eds) Agro-industries for development. Wallingford: CAB International y FAO (Food and Agricultural Organization of the United Nations). 